\title{
Molecular Docking, Drug-Likeness Studies and ADMET Prediction of Quinoline Imines for Antimalarial Activity
}

\author{
JAHNABI KALITA, DIPAK CHETIA and MITHUN RUDRAPAL*
}

Dept. of Pharmaceutical Sciences, Dibrugarh University, Dibrugarh - 786 004, Assam, India rsmrpal@gmail.com

Received 3 November 2018 / Accepted 3 December 2018

\begin{abstract}
A novel series of quinoline imines were designed by molecular manipulation approach using the principle of rational drug design. Newly designed quinoline imines were screened virtually for antimalarial effectiveness and also for drug-likeness using various in silico tools of drug design. The molecular docking was performed against $P$. falciparum parasite targeting specific cysteine protease falcipain 2 enzyme. In addition, drug-likeness and ADMET prediction studies were carried out using in silico tools. Our study reports antimalarial potential of novel quinoline imines as druglike molecules which can be further developed as potent antimalarial agents (falcipain 2 inhibitors) possibly against resistant $P$. falciparum parasite.
\end{abstract}

Keywords: P. falciparum, Resistance, Falcipain 2, Quinoline imines, Antimalarial

\section{Introduction}

Malaria continues to be a growing infectious disease burden around the world. According to WHO, about 200-300 million people are afflicted by malaria with approximately 430,000 deaths per year globally ${ }^{1}$. Plasmodium falciparum causes dreadful malaria infections such as cerebral malaria in children as well as in adults. It is responsible for most of the malariarelated deaths in humans ${ }^{2,3}$. Over the past few decades, the emergence of drug-resistant strains of $P$. falciparum has been increasing, particularly in malaria endemic regions of the world. This has limited the clinical utility of currently available antimalarial drugs and/or antimalarial drug therapy. Today, the increasing burden of resistant malaria has thus become a serious health concern in malaria control and prevention worldwide ${ }^{4-6}$.

The above challenging issue has stimulated medicinal chemists and discovery scientists to search for new antimalarial lead molecules / drug candidates as alternative therapeutic options for the treatment of resistant malaria. In this study, some novel quinoline imines were designed, modeled and screened for antimalarial effectiveness and drug-likeness assessment using various in silico tools of drug design. The molecular docking of designed quinoline imines was virtually performed against $P$. falciparum targeting specific cysteine 
protease falcipain 2 enzyme. In addition, drug-likeness screening and ADMET prediction studies were also carried out using in silico tools. Our aim was to develop quinoline imines as potent antimalarial molecules (falcipain 2 inhibitors) effective against resistant $P$. falciparum parasite.

\section{Experimental}

As a part of our ongoing research program towards developing potent antimalarial drugs, a novel series of quinoline imines were designed by molecular manipulation approach. Fifteen molecules, QI-1 to QI-15 (Figure 1) were designed with diverse substitution patterns $(\mathrm{o} / \mathrm{m} / \mathrm{p}$ - substituted aryl moiety) at the basic framework of quinoline-imine scaffold, considering pharmacodynamic importance of the quinoline imine component and structure and property parameters of the substituent relevant to biological activity.

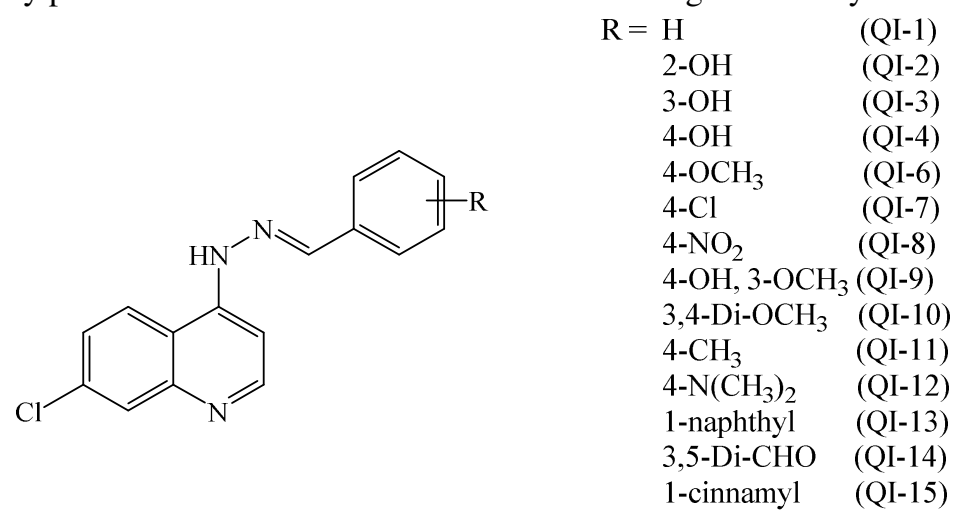

Figure 1. Designed quinoline imines

\section{Docking study}

Molecular modeling studies were carried out using Dell Precision work station T3400 running Intel Core2 Duo Processor, 4 GB RAM, 250 GB hard disk and NVidia Quodro FX 4500 graphics card. Two-dimensional (2D) structures of all compounds were built on Chemdraw Ultra 10.0 (Cambridge Soft Co., USA, 2010) and Marvin Sketch (ChemAxon LLC, Cambridge, USA, 2015) software.

Protein-ligand docking studies were carried out using biovia discovery studio (DS) $v 4.5$ (2015) software. Molecular docking was performed for all designed compounds, QI-1 to QI-15 using P. falciparum falcipain 2 enzyme. The x-ray crystal structure of falcipain 2-E64 (PDB id: 3BPF) was retrieved from the RCSB protein data bank (http://www.rcsb.org/pdb/) and Chain A of the protein determined at a resolution of $2.9 \AA$ was used in the study ${ }^{4}$. After energy minimization, the Chain A of falcipain 2 protein was defined as a receptor and the binding site sphere was selected based on the ligand binding location of E-64. A receptor grid was thereby generated around the binding cavity (active sites) of protein by specifying the key amino acid residues (Cys 42, Gly 83 and His 174) $)^{1,7}$. In DS, binding site sphere was set with a radius of $20 \AA$ and $x, y, z$ dimensions of -52.25 , $4.46,-19.25$, respectively. Flexible molecular docking was performed where the protein was held rigid, while the ligands were allowed to be flexible during refinement. During docking, the falcipain 2-E-64 complex was imported and E-64 molecule (co-crystal ligand) was removed, and ligands were placed in the predicted binding site (grid box). Docking was performed using the dock ligands module of LibDock genetic algorithm program of DS. 
All docking and consequent scoring parameters used were kept at their default settings. The LibDock scores of the docked ligands were calculated. Different dock poses were studied to know the best binding mode of receptor-ligand complex in terms of scoring function. All docked poses were scored, ranked and the best pose of each compound having the highest score was identified. The best docked pose was later used for the receptor-ligand interaction analysis. Interaction of ligands with receptor was studied to know the best binding orientation of receptor-ligand complex having maximum LibDock score. Binding modes of the best pose for each compound was also analyzed with the help of 3D receptor-ligand complex. Different non-bonding interactions (hydrogen bonding and hydrophobic) were also analyzed with the help of 2D diagram of docked receptor-ligand complexes.

\section{Drug-likeness studies}

In silico calculations of the molecular properties and drug-likeness parameters for all compounds, QI-1 to QI-15 was performed based on theoretical approaches to identify the compounds which violate the optimum requirements for drug-likeness. Molecular properties (molecular weight, LogP value, number of hydrogen bond acceptor(s), number of hydrogen bond donor(s), total polar surface area) incorporated in Lipinski's rule of five ${ }^{12}$ and other physicochemical parameters like aqueous solubility $(\operatorname{LogS})$, molar refractivity and molar volume were calculated using Calculation of Molecular Properties module of Biovia DS $v$ 4.5 software. The number of rotatable bonds was predicted using Molsoft Online software (http://www.molsoft.com/, 2016) and non-violation of drug-likeness was calculated using Molinspiration online software (http://www.molinspiration.com/, 2016) ${ }^{1,8-10}$.

\section{ADMET prediction}

ADME-Toxicity (ADMET) for all the compounds was calculated in silico using ADMET descriptor module of Biovia DS $v 4.5$ software. Six mathematical models (aqueous solubility, blood-brain barrier penetration, cytochrome P450 2D6 inhibition, hepatotoxicity, human intestinal absorption and plasma protein binding) were used to quantitatively predict properties related to ADMET characteristics or pharmacokinetics of molecules ${ }^{1,11}$.

\section{Results and Discussion}

Designing quinoline imines

The 4-aminoquinoline scaffold is considered as the key requirement for antimalarial activity for quinoline-based antimalarial agents. The importance of 4-aminoquinoline scaffold incorporated with hydrazyl moiety as antimalarial component has been reported. Substitution of quinoline hydrazine moiety with aryl/substituted aryl group could lead to our target quinoline-imine conjugate with diverse substitutions.

\section{Docking study}

Docking is generally used to find the best binding modes as well as orientation of small molecules (ligands) bound to a protein molecule in order to predict their binding affinity as well as potential of biological activity ${ }^{12}$. In our study, 3D structure of falcipain 2 protein molecule was used as possible antimalarial drug target for quinoline imines. The protein model used for docking study was validated as depicted below.

The 3D crystal structure of falcipain 2 co-crystallized with the inhibitor transepoxysuccinyl- $L$-leucylamido-(4-guanidino) butane (E-64) with active site (receptor grid model) defined by Cys 42, Gly 83 and His 174 residues was optimized and used for the study. 
The co-crystal structure of falcipain 2-E-64 complex and the receptor grid model used for docking are presented in Figure 2. The co-crystallized ligand, E-64 was re-docked using flexible docking simulations (LibDock module of DS) into the original structure of the target protein, falcipain-2 by non-covalent docking method. For this study, docking parameters were set to the software's default values. E-64 was successfully re-docked to the predicted active sites of falcipain 2 with an acceptable RMSD value of $1.124 \AA$ A. Further, in order to reproduce an experimentally observed ligand-binding mode, the co-crystallized ligand, E-64 (a selective falcipain 2 inhibitor) was used as reference ligand. Results confirmed experimental binding conformations of E-64 in the binding pocket of receptor molecule (Figure 3).

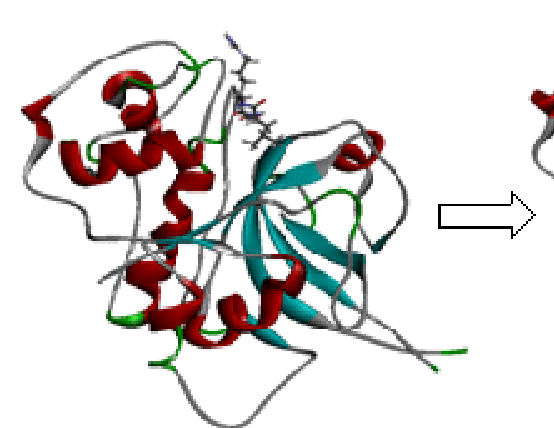

(a)

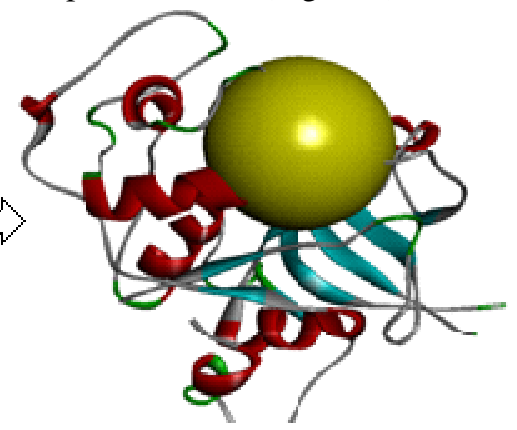

(b)

Figure 2. (a) Optimized co-crystal structure of falcipain 2 (Chain A)-E-64, (b) Receptor grid for docking

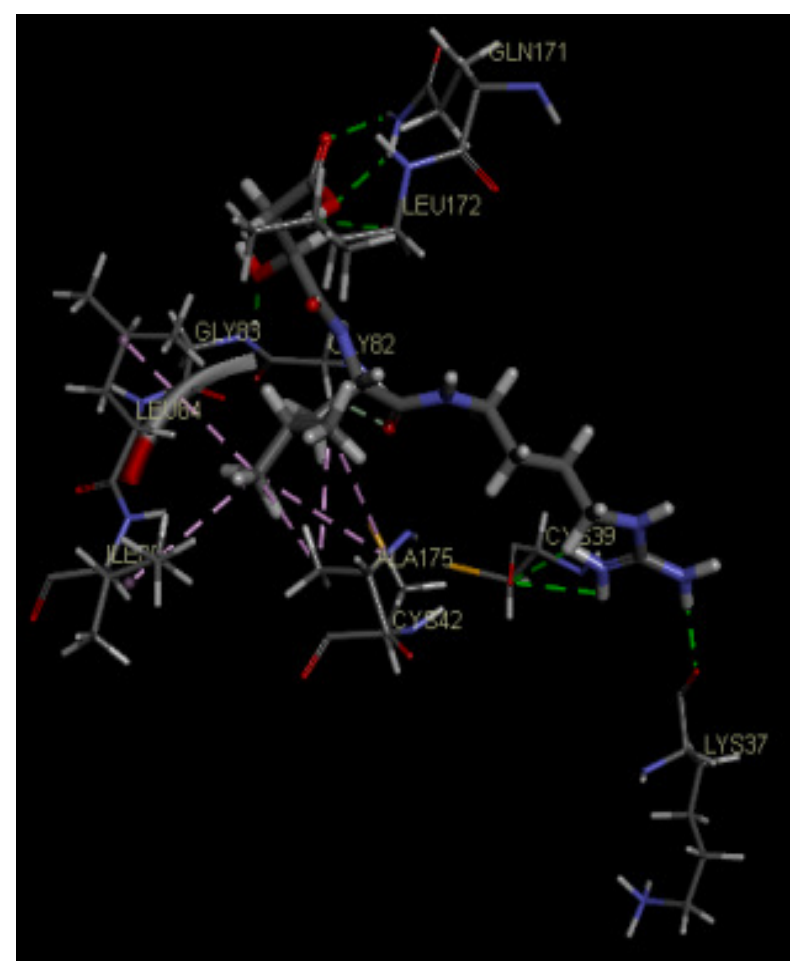




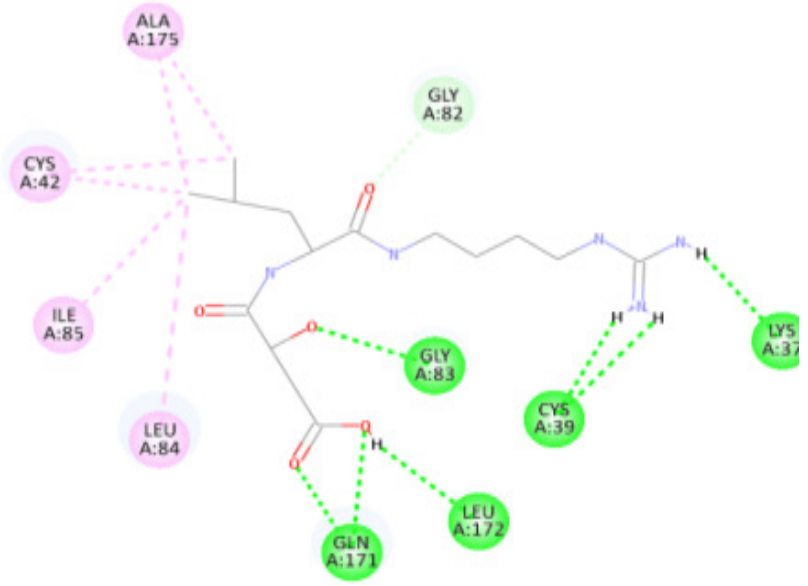

(a)
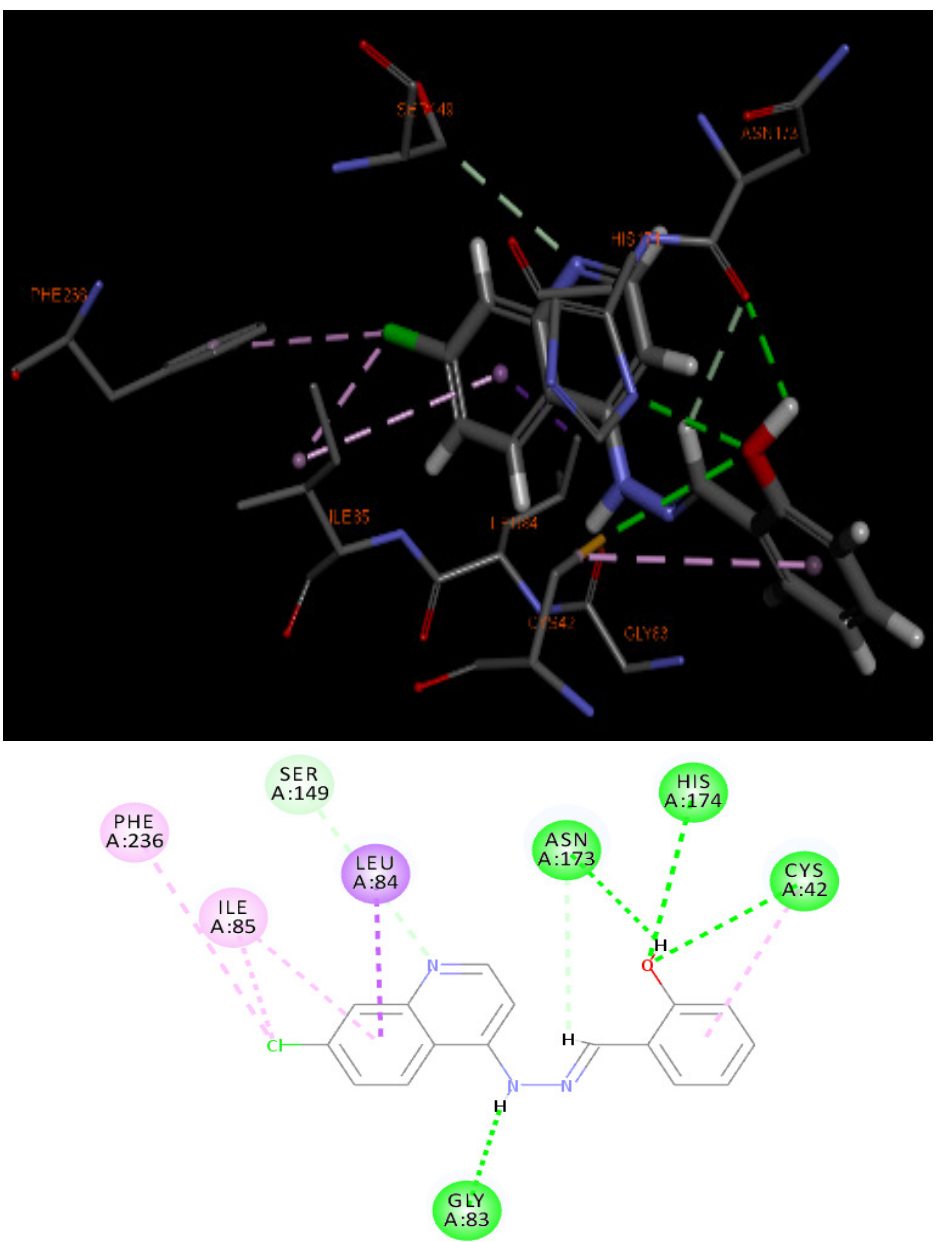

(b) 

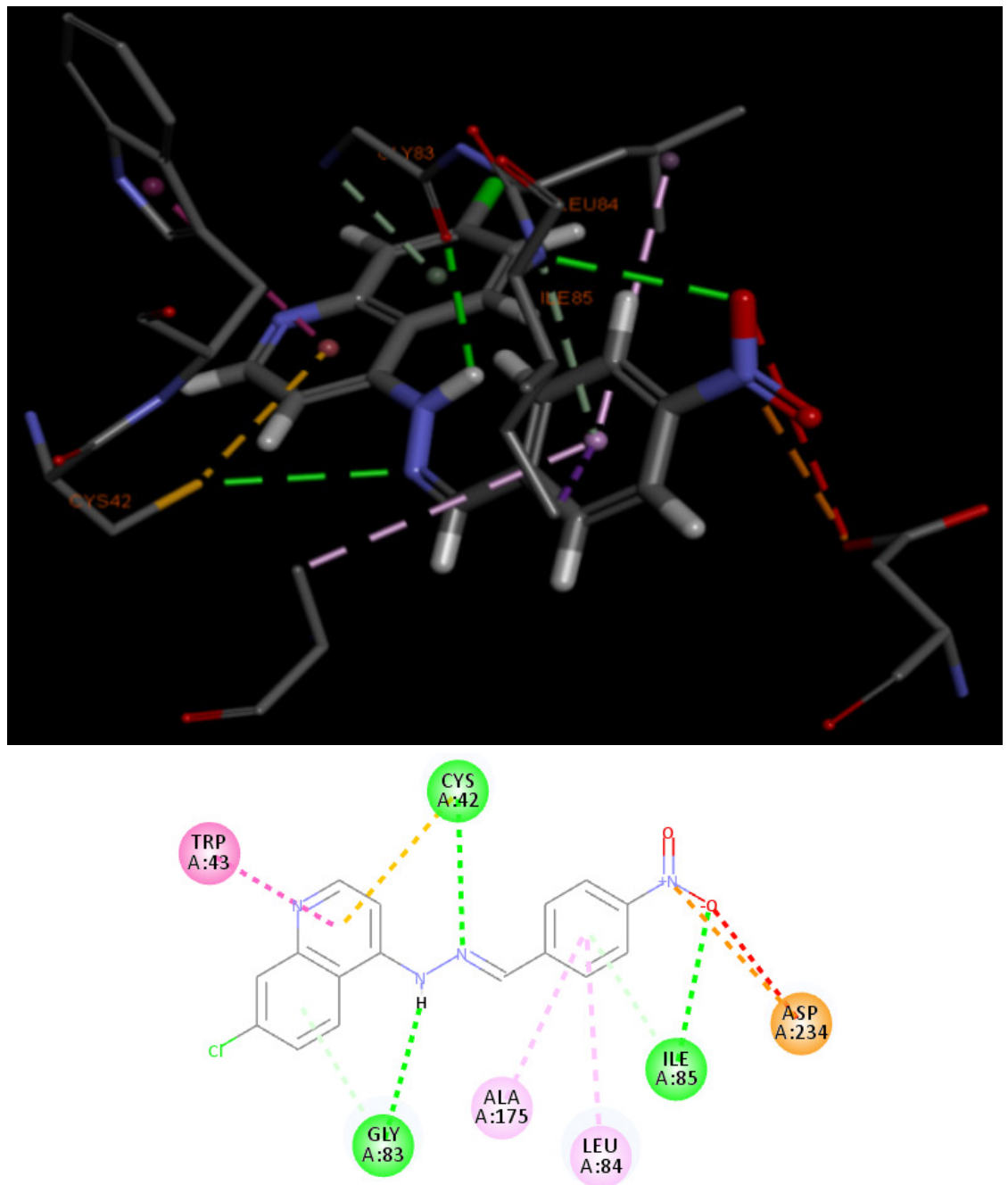

(c)

Figure 3. (a) Redocked conformer of E-64 in the active site of the protein falcipain 2 (left) and $2 \mathrm{D}$ representation of the binding interaction (right); (b) and (c): Binding mode (left) and 2D receptor-ligand interaction diagram (right) of compounds, QI-2 and QI-8 at binding pocket of falcipain 2 (left), respectively

Docking results reveal that LibDock program successfully docked all quinoline imines into the binding pocket of falcipain 2 enzyme. All compounds could bind with the active site of falcipain-2 with high docking score (LibDock) and binding affinities in the range of 90.28 to 112.34 . The LibDock scores are depicted in Table 1. LibDock score is useful to assess the antimalarial potential of ligands as $P$. falciparum falcipain 2 inhibitors. Docking rationalizes finding new lead molecules and also gives an insight into structure-activity relationships and mode(s) of drug action based on scoring function and further analysis of binding modes from ligand-protein binding/interaction studies. 
Table 1. LibDock scores and no. of H-bonds

\begin{tabular}{ccc}
\hline Comp. code & Libdock score & No. of H-bond(s) \\
\hline QI-1 & 90.287 & 3 \\
QI-2 & 112.34 & 4 \\
QI-3 & 94.276 & 3 \\
QI-4 & 90.870 & 2 \\
QI-5 & 95.614 & 2 \\
QI-6 & 91.749 & 3 \\
QI-7 & 90.992 & 1 \\
QI-8 & 95.650 & 3 \\
QI-9 & 97.658 & 3 \\
QI-10 & 95.881 & 5 \\
QI-11 & 90.989 & 3 \\
QI-12 & 105.96 & 4 \\
QI-13 & 108.08 & 4 \\
QI-14 & 97.704 & 2 \\
QI-15 & 98.182 & 1 \\
\hline
\end{tabular}

Protein-ligand docking was performed to generate the bioactive binding poses of designed inhibitors in the active site of falcipain 2 enzyme. The 3D poses of bound ligands were visualized which reveal the best orientation of the ligand relative to the receptor as well as the conformation of the ligand and receptor (best fit of ligand in the receptor molecule). Analysis of 2D diagram indicated that various non-bonded interactions mainly polar hydrogen bonding interactions were involved between binding site residues (active site amino acids) and ligand moieties/atoms. Table 1 also depicts the number of hydrogen bonds (H-bonds) for all docked compounds. Higher the number of hydrogen bonds, higher is the binding affinity.

Table 2 reveal hydrogen bonding interactions of five most potent compounds (QI-2, QI-8, QI-10, QI-12 and QI-13) with active site residues such as Glu 36, Cys 42, Gly 40, Gly 83, Ile 85, Ser 149, Leu 172, Asn 173, His 174 and Asp 234. Compound QI-2 which showed the highest antimalarial activity with LibDock score of 112.34 could bind the active sites of falcipain-2 mainly by hydrogen bonding interactions. The 3D binding modes and 2D interaction diagrams of two most potent compounds, QI-2 and QI-8 are shown in Figure 3. Compound QI-2 formed four strong H-bonds with residues like Cys $42(\mathrm{O} \cdot \mathrm{H} \cdot \mathrm{S})$, His 174 $(\mathrm{O} \cdot \mathrm{H} \cdot \mathrm{N})$, Asn $173(\mathrm{O} \cdot \mathrm{H} \cdot \mathrm{O})$ and Gly $83(\mathrm{O} \cdot \mathrm{H} \cdot \mathrm{O})$. Three bonds were observed with residues like His $174(\mathrm{O} \cdot \mathrm{H} \cdot \mathrm{O})$, Gly $83(\mathrm{O} \cdot \mathrm{H} \cdot \mathrm{N})$ and Ser $149(\mathrm{O} \cdot \mathrm{H} \cdot \mathrm{O})$ for compound QI-8. Analysis of best docking poses reveal that the quinoline scaffold was oriented in the binding cavity (active site residues) of falcipain 2 receptor molecule. In 2D diagram, the quinoline imine moiety could occupy the binding sites of falcipain- 2 through strong $\mathrm{H}$ bonding interactions along with hydrophobic interactions. Such interactions afforded good stability between receptor and ligand molecules. Further analysis of docking interactions, it was found that quinoline ring played a crucial role in protein-ligand binding. Substituents increased binding strength by forming additional $\mathrm{H}$-bonds that facilitated much stronger interaction of ligands with the receptor (falcipain-2 protein) molecule with optimum binding affinity to achieve desired antimalarial activity. 
Table 2. Details of hydrogen bonding for most active ligands

\begin{tabular}{|c|c|c|c|c|c|c|c|}
\hline \multirow{2}{*}{$\begin{array}{l}\text { Comp. } \\
\text { Code }\end{array}$} & \multirow{2}{*}{ H-bond(s) } & \multicolumn{2}{|c|}{ H-binding ligand } & \multicolumn{3}{|c|}{ H-binding receptor } & \multirow{2}{*}{$\begin{array}{c}\text { H-bond } \\
\text { distance }\left(\mathrm{A}^{\mathrm{o}}\right)\end{array}$} \\
\hline & & Element & Type & Residue & Element & Type & \\
\hline \multirow{4}{*}{ QI-2 } & \multirow{4}{*}{4} & $\mathrm{O}$ & A & Cys 42 & $\mathrm{~S}$ & $\mathrm{D}$ & 3.411 \\
\hline & & $\mathrm{O}$ & A & His 174 & $\mathrm{~N}$ & D & 3.399 \\
\hline & & $\mathrm{H}$ & A & Asn 173 & $\mathrm{O}$ & D & 2.430 \\
\hline & & $\mathrm{H}$ & A & Gly 83 & $\mathrm{O}$ & D & 2.142 \\
\hline \multirow{3}{*}{ QI-8 } & \multirow{3}{*}{3} & $\mathrm{O}$ & A & His 174 & $\mathrm{H}$ & $\mathrm{D}$ & 2.925 \\
\hline & & $\mathrm{H}$ & A & Gly 83 & $\mathrm{~N}$ & $\mathrm{D}$ & 2.674 \\
\hline & & $\mathrm{O}$ & A & Ser 149 & $\mathrm{H}$ & $\mathrm{D}$ & 3.128 \\
\hline \multirow[t]{6}{*}{ QI-10 } & \multirow{5}{*}{5} & $\mathrm{O}$ & A & Cys 42 & $S$ & D & 2.632 \\
\hline & & $\mathrm{O}$ & A & Ile 85 & $\mathrm{~N}$ & D & 2.840 \\
\hline & & $\mathrm{H}$ & A & Gly 83 & $\mathrm{O}$ & D & 2.220 \\
\hline & & $\mathrm{H}$ & A & Asp 234 & $\mathrm{O}$ & D & 3.009 \\
\hline & & $\mathrm{H}$ & A & Gly40 & $\mathrm{O}$ & D & 2.530 \\
\hline & \multirow{4}{*}{4} & $\mathrm{O}$ & A & Glu 36 & $\mathrm{~N}$ & D & 3.208 \\
\hline \multirow{4}{*}{ QI-12 } & & $\mathrm{N}$ & $\mathrm{D}$ & Cys 42 & $\mathrm{O}$ & A & 3.266 \\
\hline & & $\mathrm{H}$ & A & Asn 173 & $\mathrm{O}$ & D & 2.175 \\
\hline & & $\mathrm{H}$ & A & Cys 42 & $S$ & $\mathrm{D}$ & 3.543 \\
\hline & \multirow{4}{*}{4} & $\mathrm{H}$ & A & His 174 & $\mathrm{~N}$ & $\mathrm{D}$ & 4.084 \\
\hline \multirow{3}{*}{ QI-13 } & & $\mathrm{H}$ & A & Cys 42 & $S$ & $\mathrm{D}$ & 3.381 \\
\hline & & $\mathrm{H}$ & A & Leu 172 & $\mathrm{O}$ & D & 2.523 \\
\hline & & $\mathrm{H}$ & A & Asn 173 & $\mathrm{O}$ & $\mathrm{D}$ & 2.546 \\
\hline
\end{tabular}

\section{Drug-likeness}

The results of predicted Lipinski's parameters and drug-likeness parameters of the quinoline imines, QI-1 to QI-15 are depicted in Table 3. Results reveal that all the compounds possess good drug-like properties based on Lipinski's rule of five ${ }^{13}$ with additional parameters such as molar refractivity (MR) and number of rotable bonds (nRotB). All compounds obeyed Lipinski's rule of five and Veber rule. Lipinski rule of five is a rule to evaluate drug likeness to determine if a chemical compound has a certain pharmacological or biological activity to make it an orally active drug ${ }^{4}$. In our study, compounds did not violate Lipinski rule of five parameters. Poor absorption or permeation of a ligand is more likely if a drug-like molecule have more than one of five rule violations. Values of $\log$ P, MW and TPSA indicated that compounds possessed good membrane permeability and oral bioavailability, whereas, nRotb bonds suggested that compounds had good intestinal availability. MR values were also found in permissible range which indicated good oral bioavailaility for all the compounds. Hydrophobicity, membrane permeability and bioavailability are dependent on molecule's MW, LogP, HBA and HBD. Molecules violating more than one of these rules fail to exhibit optimum bioavailability. Number of rotatable bonds is important for molecular conformational studies (i.e., stereoselectivity of drug molecules) for optimal binding with the receptor molecule. Further, TPSA and MR are also useful parameters for drug's transport and biodistribution ${ }^{8,9}$. The drug score combines drug-likeness, lipophilicity, solubility, molecular weight and the risk of toxicity into a single numerical value that can be used to predict a global value for each compound as a potential new drug candidate ${ }^{6}$. The overall analysis of drug-likeness studies strongly suggest that newly designed quinoline imines possess good drug-likeness behavior favorable for optimal membrane permeability, transport and bioavailability and eventual interaction with the receptor molecule. 
Table 3. Drug-likeness properties

\begin{tabular}{ccccccccccc}
\hline $\begin{array}{c}\text { Comp. } \\
\text { Code }\end{array}$ & MW & LogP & nHBA & nHBD & $\begin{array}{c}\text { TPSA } \\
\left(\mathrm{A}^{2}\right)\end{array}$ & nViolations & MS & $\begin{array}{c}\text { MV } \\
\left(\mathrm{A}^{3}\right)\end{array}$ & nRotB \\
\hline QI-1 & 281.74 & 4.299 & 3 & 1 & 37.28 & 0 & -6.07 & 252.27 & 3 \\
QI-2 & 297.74 & 4.005 & 4 & 2 & 57.51 & 0 & -5.52 & 262.80 & 3 \\
QI-3 & 297.74 & 4.057 & 4 & 2 & 57.51 & 0 & -5.58 & 262.89 & 3 \\
QI-4 & 297.74 & 4.057 & 4 & 2 & 46.51 & 0 & -5.62 & 262.82 & 3 \\
QI-5 & 311.77 & 4.283 & 4 & 1 & 46.51 & 0 & -6.21 & 284.19 & 4 \\
QI-6 & 311.77 & 4.283 & 4 & 1 & 37.28 & 0 & -6.23 & 284.12 & 4 \\
QI-7 & 316.19 & 4.963 & 3 & 1 & 83.10 & 0 & -6.85 & 269.46 & 3 \\
QI-8 & 326.74 & 4.193 & 5 & 1 & 66.74 & 0 & -6.46 & 290.45 & 4 \\
QI-9 & 327.77 & 4.041 & 5 & 2 & 55.74 & 0 & -5.69 & 295.54 & 4 \\
QI-10 & 341.80 & 4.266 & 5 & 1 & 37.28 & 0 & -6.31 & 315.54 & 5 \\
QI-11 & 281.74 & 4.785 & 3 & 1 & 37.28 & 0 & -6.60 & 273.21 & 3 \\
QI-12 & 307.78 & 4.659 & 3 & 1 & 40.52 & 0 & -7.03 & 293.58 & 4 \\
QI-13 & 324.81 & 4.461 & 4 & 1 & 37.28 & 0 & -6.04 & 301.82 & 4 \\
QI-14 & 331.80 & 5.207 & 3 & 1 & 49.78 & 1 & -7.91 & 301.09 & 3 \\
QI-15 & 309.75 & 4.058 & 4 & 1 & 54.35 & 0 & -6.26 & 280.53 & 4 \\
\hline
\end{tabular}

MW- Molecular weight, LogP-Log of octanol/water partition coefficient, nHBA- No. of hydrogen bond accepror(s), nHBD- No. of hydrogen bond donor(s), TPSA-Total polar surface area, nViolations- No. of rule of five violations, MS- Molar aqueous solubility, MR-Molar refractivity, MVMolar volume, $n$ RotB- No. of rotable bonds

Table 4. Theoretical ADMET parameters

\begin{tabular}{ccccccc}
\hline $\begin{array}{c}\text { Comp. } \\
\text { code }\end{array}$ & $\begin{array}{c}\text { Aqueous } \\
\text { solubility }\end{array}$ & $\begin{array}{c}\text { BBB } \\
\text { penetration }\end{array}$ & $\begin{array}{c}\text { CYP P450 } \\
\text { 2D6 } \\
\text { inhibition }\end{array}$ & Hepatotoxicity & $\begin{array}{c}\text { Intestinal } \\
\text { absorption }\end{array}$ & PP binding \\
\hline QI-1 & 2 & 1 & TRUE & TRUE & 0 & TRUE \\
QI-2 & 2 & 1 & TRUE & TRUE & 0 & TRUE \\
QI-3 & 2 & 1 & TRUE & TRUE & 0 & TRUE \\
QI-4 & 2 & 1 & TRUE & TRUE & 0 & TRUE \\
QI-5 & 2 & 1 & TRUE & TRUE & 0 & TRUE \\
QI-6 & 2 & 1 & TRUE & TRUE & 0 & TRUE \\
QI-7 & 1 & 0 & TRUE & TRUE & 0 & TRUE \\
QI-8 & 2 & 2 & TRUE & TRUE & 0 & TRUE \\
QI-9 & 2 & 1 & TRUE & TRUE & 0 & TRUE \\
QI-10 & 2 & 1 & TRUE & TRUE & 0 & TRUE \\
QI-11 & 2 & 0 & TRUE & TRUE & 0 & TRUE \\
QI-12 & 2 & 0 & TRUE & TRUE & 0 & TRUE \\
QI-13 & 2 & 1 & TRUE & TRUE & 0 & TRUE \\
QI-14 & 1 & 0 & TRUE & TRUE & 0 & TRUE \\
QI-15 & 2 & 1 & TRUE & TRUE & 0 & TRUE \\
\hline
\end{tabular}

Aqueous solubility: 3-Good, 2-Low; BBB (Blood brain barrier) penetration: 3-Low, 2-Medium, 1Moderate; Cytochrome (CYP) P450 2D6 inhibition: False-Non-inhibitor; Hepatotoxicity: True-Toxic, False-Non-toxic; Intestinal absorption: 0-Good; Plasma protein (PP) binding: True-Highly bounded, False-Poorly bounded 


\section{ADMET prediction}

The ADMET values of newly designed quinoline imines presented in Table 4 were found in acceptable range with favorable ADMET properties. ADMET (absorption, distribution, metabolism, excretion and toxicity) properties have a predictable influence on pharmacokinetic and pharmacodynamic effects of drug molecules. The calculation of ADMET properties is therefore essential towards optimizing the new drug molecules. These properties influence oral bioavailability, cell permeation and metabolism of drug molecules ${ }^{14}$.

All the compounds were predicted to have good intestinal absorption and non-inhibitors of cytochrome P450 2D6 (CYP2D6) with medium to moderate blood-brain barrier (BBB) penetration. $\mathrm{BBB}$ penetration is mandatory for the drug to be used in the treatment of cerebral malaria. The CYP2D6 enzyme is one of the important enzymes involved in drug metabolism. The aqueous solubility prediction (defined in water at $25^{\circ} \mathrm{C}$ ) indicated that most of the compounds were soluble in water. The predictive hepatotoxicity was observed for a few compounds among three series. Some of the compounds were found to be highly bound with plasma protein, while some were poorly bound with plasma protein ${ }^{14}$.

\section{Conclusion}

Newly designed quinoline imines are reported to be potent antimalarial molecules as possible $P$. falciparum falcipain 2 inhibitors. Molecular docking study and in silico druglikeness and ADMET prediction studies confirmed the antimalarial potential and druglikeness of newer quinoline imines. Based upon our present work, future work could be directed towards further molecular optimization of quinoline imines based upon SAR and QSAR studies. This strategy would lead to the development of these novel quinoline imines as future antimalarial agents.

\section{Acknowledgement}

Authors are thankful to the Head, Department of Pharmaceutical Sciences, Dibrugarh University, Dibrugarh, Assam (India) for providing necessary facilities to carry out in silico studies of compounds.

\section{References}

1. Rudrapal M, Chetia D and Singh V, J Enzyme Inhib Med Chem., 2017, 32(1), 11591173; DOI:10.1080/14756366.2017.1363742

2. Medhi A, Chetia D and Rudrapal M, Indian J Pharm Edu Res., 2018, 52(3), 472-479.

3. Rudrapal M and Chetia D, Drug Des Devl Ther., 2016, 10, 3575-3590; DOI:10.2147/DDDT.S118116

4. Rudrapal M, Banu Z W and Chetia D, Med Chem Res., 2018, 27(2), 653-668; DOI:10.1007\%2Fs00044-017-2090-8

5. Rudrapal M, Chetia D and Prakash A, Med Chem Res., 2013, 22(8), 3703-3711; DOI:10.1007\%2Fs00044-012-0371-9

6. Roy S, Chetia D, Rudrapal M and Prakash A, Med Chem., 2013, 9, 379-383; DOI:10.2174/1573406411309030008

7. Sashidhara K V, Kumar K, Dodda R P, Krishna N N, Agarwal P, Srivastava K and Puri S K, Bioorg Med Chem., 2012, 22(12), 3926-3930;

DOI:10.1016/j.bmcl.2012.04.100

8. Sharma D, Chetia D and Rudrapal M, Asian J Chem., 2016, 28(4), 782-788; DOI:10.14233/ajchem.2016.19478 
9. Kashyap A, Chetia D and Rudrapal M, Indian J Pharm Sci., 2016, 78, 892-911.

10. Gogoi J, Chetia D, Kumawat M K and Rudrapal M, Indian J Pharm Edu Res., 2016, 50(4), 591-597; DOI:10.5530/ijper.50.4

11. Faidallah H M, Al-Mohammadi M M, Alamry K A and Khan K A, J Enzyme Inhib Med Chem., 2016, 31(supp1), 157-163; DOI:10.1080/14756366.2016.1180594

12. Kitchen D B, Decornez H, Furr J R and Bajorath J, Nat Rev Drug Discov., 2004, 3(11), 935-949.

13. Lipinski C A, Lombardo F, Dominy B W and Feeney P J, Adv Drug Deliv Rev., 1997, 23(1-3), 3-26; DOI:10.1016/S0169-409X(96)00423-1

14. Singh S and Srivastava P, Comput Mol Biosci., 2015, 5, 20-33;

DOI: $10.4236 / \mathrm{cmb} .2015 .52004$ 